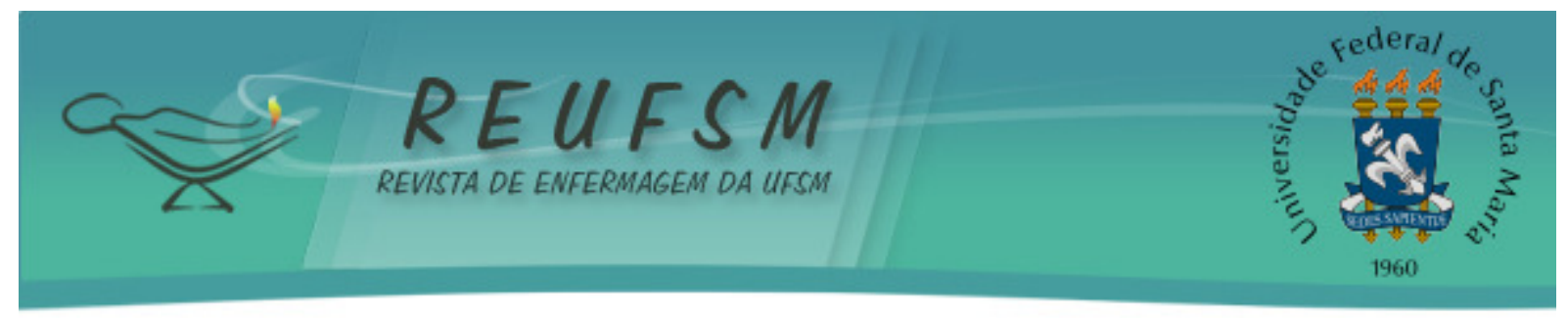

\title{
DISTÚRBIOS PSÍQUICOS MENORES EM ESTUDANTES DE GRADUAÇÃO EM ENFERMAGEM: ESTUDO TRANSVERSAL
}

\section{MINOR PSYCHIATRIC DISORDERS IN NURSING UNDERGRADUATES STUDENTS: A CROSS-SECTIONAL STUDY}

\section{DISTURBIOS PSÍQUICOS MENORES EN ESTUDIANTES DE GRADUACIÓN EN ENFERMERÍA: ESTUDIO TRANSVERSAL}

\author{
Cecília Mariane Pinheiro Pedro ${ }^{1}$ \\ Tânia Solange Bosi de Souza Magnago ${ }^{2}$ \\ Cíntia da Silva Marconato ${ }^{3}$ \\ Bruna Xavier Morais ${ }^{4}$ \\ Ana Carolina de Souza Magnago ${ }^{5}$ \\ Rafaela Andolhe 6
}

Doi: $10.5902 / 2179769224949$

RESUMO: Objetivo: Verificar a prevalência e os fatores associados aos Distúrbios Psíquicos Menores (DPMs) em estudantes de graduação em enfermagem de uma universidade pública do Rio Grande do Sul, Brasil. Método: Estudo transversal, desenvolvido em dezembro de 2014, com 149 estudantes de graduação em enfermagem. Utilizou-se questionário autopreenchível com questões sociodemográficas, acadêmicas e de saúde e a versão brasileira do Self Reporting Questionnaire - 20. Para a análise, utilizou-se a estatística descritiva e analítica no programa Predictive Analytics Software (PASW Statistics $\left.{ }^{\circledR}\right)$. Resultados: Predominaram estudantes do sexo feminino, com idade entre 21 e 30 anos, solteiros e que estavam cursando o $4^{\circ}$ semestre da graduação. A prevalência de DPMs foi de 54,4\%. Dos fatores avaliados, ter o diagnóstico médico de alguma patologia foi significativo para DPMs. Conclusão: A alta prevalência de DPMs evidencia a necessidade de acompanhamento e de planejamento de estratégias de prevenção do sofrimento psíquico nessa população.

Descritores: Estudantes de enfermagem; Enfermagem; Estresse psicológico; Sintomas psíquicos; Estudos transversais.

ABSTRACT: Aim: to verify the prevalence and the factors associated to the Minor Psychiatric Disorders (MPD) in nursing undergraduate students of a public University in Rio Grande do Sul, Brazil. Method: cross-sectional study, developed in December 2014, with 149 nursing students. A self-administered questionnaire was used with sociodemographic, academic and health questions and the Brazilian version of the Self Reporting Questionnaire - 20. For the analysis, the descriptive

\footnotetext{
${ }^{1}$ Enfermeira. Mestranda em enfermagem pela Universidade Federal de Santa Maria. Santa Maria, RS, Brasil. Email: cecilia-mariane@ hotmail.com

2 Enfermeira. Doutora em enfermagem. Professora adjunta na Universidade Federal de Santa Maria. Santa Maria, RS, Brasil. E-mail: tmagnago@ terra.com.br

${ }^{3}$ Enfermeira. Mestre em enfermagem. Hospital Universitário de Santa Maria. Santa Maria, RS, Brasil. E-mail: cintiamarconato@gmail.com

${ }^{4}$ Enfermeira. Mestranda em enfermagem pela Universidade Federal de Santa Maria. Santa Maria, RS, Brasil. Email: bruna_morais100@hotmail.com

5 Acadêmica do Curso de psicologia. Universidade Federal de Santa Maria. Santa Maria, RS, Brasil. E-mail: anasmagnago@gmail.com

${ }^{6}$ Enfermeira. Doutora em enfermagem. Professora adjunta na Universidade Federal de Santa Maria. Santa Maria, RS, Brasil. E-mail: rafaela.andolhe1@gmail.com
} 


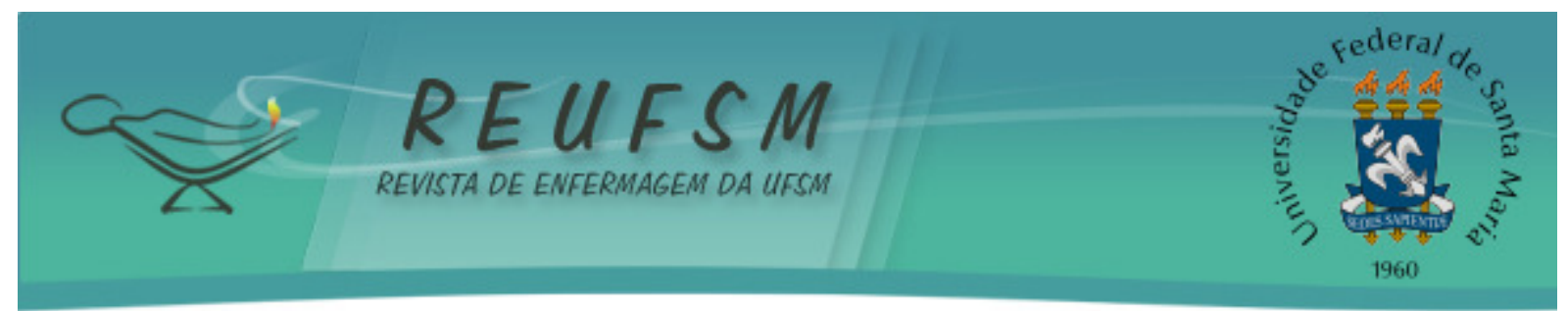

and analytical statistics were used in the Predictive Analytics Software (PASW Statistics $®)$. Results: Female students, aged between 21 and 30, who were single and on the fourth semester of the graduation course were predominant. The MPD prevalence was $54.4 \%$. Among the assessed factors, having the medical diagnosis for some pathology was significant for MPD. Conclusion: the elevated prevalence of MPD, stresses the necessity of attending and planning prevention strategies for the psychological distress in this population.

Descriptors: Students, nursing; Nursing; Stress, Psychological; Psychic symptoms; Crosssectional studies.

RESUMEN: Objetivo: verificar la predominancia y los factores asociados a los disturbios psíquicos menores (DPMs) en estudiantes de graduación en enfermería de una Universidad Pública de Rio Grande do Sul, Brasil. Método: estudio transversal, desarrollado en diciembre de 2014, con 149 estudiantes de graduación en enfermería. Se utilizó cuestionario autoadministrado con cuestiones sociodemográficas, académicas y de salud y la versión brasileña del Self Reporting Questionnaire - 20. Para el análisis se utilizó estadística descriptiva y analítica en el programa Predictive Analytics Software (PASW Statistics $\left.{ }^{\circledR}\right)$. Resultados: predominó estudiantes del sexo femenino, con edades entre 21 y 30 años, solteros y que estaban en el cuarto semestre de la graduación. La predominancia de los DPMs fue de 54,4\%. Entre los factores evaluados, tener el diagnóstico médico de una patología fue significativa para los DPMs. Conclusión: la alta incidencia de los DPM destaca la necesidad del control y planificación de estrategias de prevención de los trastornos psíquicos en esta población.

Descriptores: Estudiantes de enfermería; Enfermería; Estrés psicológico; Síntomas psíquicos; Estudio transversales.

\section{INTRODUÇÃO}

As exigências do universo acadêmico, como as novas rotinas de atividades, a saída da casa dos pais, o número de disciplinas e as aulas em turno integral podem desencadear uma confusão de sentimentos no estudante universitário. Ao mesmo tempo em que se encontra satisfeito com a oportunidade de ensino, pode sentir-se frustrado ao se deparar com as exigências do curso escolhido. ${ }^{1}$ Dentre os cursos de graduação, o de enfermagem tem sido considerado como um dos mais exaustivos. A convivência diária com situações de intenso sofrimento humano e a responsabilidade pela vida e saúde dos indivíduos envolvem algumas das peculiaridades inerentes à profissão de enfermagem, e podem favorecer o adoecimento psíquico no estudante. $^{2}$

Algumas situações, no decorrer da graduação, podem gerar insegurança e receio nos estudantes, como a adaptação ao ambiente de aulas práticas e estágios; o relacionamento com novos colegas e professores; os primeiros contatos com a morte e o sofrimento de pacientes e familiares; a realização dos primeiros procedimentos e o medo de contrair doenças; a exposição 


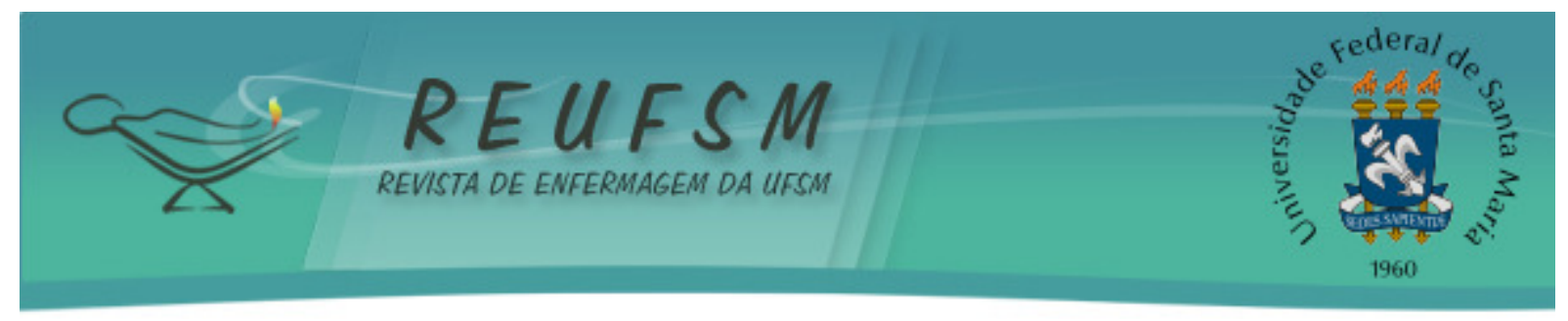

aos riscos ambientais (físicos, químicos, biológicos, ergométricos, psíquicos e de acidentes de trabalho); a construção de trabalhos acadêmicos e preocupações com avaliações. ${ }^{1,3}$

Essas demandas podem favorecer o surgimento de Transtornos Mentais nos estudantes de enfermagem, como é o caso dos Distúrbios Psíquicos Menores (DPMs), objeto deste estudo. Estes consistem em transtornos mentais menos graves, caracterizados por um conjunto de sintomas como a ansiedade, insônia, fadiga, redução da concentração, irritabilidade, redução da capacidade funcional e somatização. ${ }^{4}$ Eles podem estar relacionados com estressores presentes na vida pessoal e/ou profissional, como a redução de apoio social, condições de vida e de trabalho ruins, baixa renda e baixa escolaridade. ${ }^{5}$ Entre profissionais de enfermagem, os DPMs também são descritos em estudos ${ }^{6-7}$ e as prevalências variam entre $20,6 \%$ e $35 \%$. Tais resultados evidenciam a enfermagem como uma profissão que, por suas características, pode favorecer o adoecimento psíquico no trabalhador.

No entanto, o adoecimento pode ter início nos primeiros contatos com a profissão, ainda na formação dos graduandos de enfermagem. Se não identificado e tratado, pode ser agravado no desenrolar da vida ocupacional. Em vista disso, justifica-se o desenvolvimento deste estudo pela possibilidade de identificação da prevalência desse agravo em estudantes de graduação em enfermagem. Os achados do estudo podem subsidiar as coordenações de cursos e demais órgãos das instituições de ensino no desenvolvimento de ações de prevenção e promoção à saúde mental dos universitários. Acredita-se que a redução dos problemas de saúde no início da vida profissional contribui para a maior satisfação no trabalho, bem como para a redução de futuros problemas de saúde relacionados ao mesmo. Outro aspecto importante, que também justifica a realização do estudo, relaciona-se a escassez de investigações sobre DPMs na população universitária. ${ }^{8}$

Portanto, diante das particularidades laborais que envolvem a enfermagem e dos possíveis desafios que os futuros profissionais enfrentam durante a graduação, este estudo, norteado pela questão de pesquisa: "Qual a prevalência e os fatores associados aos Distúrbios Psíquicos Menores em estudantes de graduação em enfermagem?", objetivou verificar a prevalência e os fatores associados aos Distúrbios Psíquicos Menores em estudantes de graduação em enfermagem de uma universidade pública do Rio Grande do Sul, Brasil. 


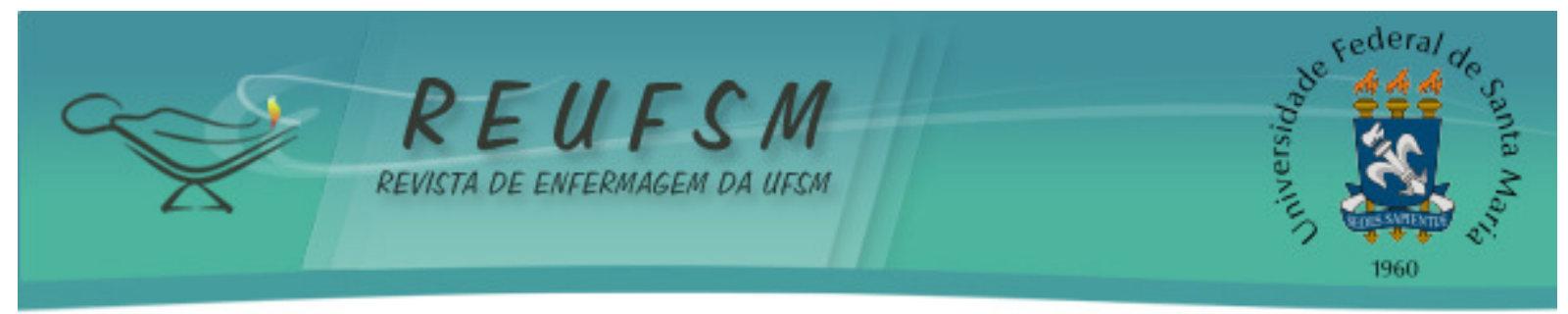

\section{MÉTODO}

Estudo transversal, desenvolvido com 149 dos 197 estudantes matriculados no Curso de Graduação em Enfermagem do Centro de Ciências da Saúde, da Universidade Federal de Santa Maria (UFSM). Optou-se por realizar a pesquisa com a população total de estudantes, visto que a mesma constituiu um número acessível de estudantes para a coleta de dados. Mesmo assim, foi realizado cálculo amostral por meio da seguinte fórmula: ${ }^{9}$

$$
n=\frac{Z_{\alpha / 2}^{2} \cdot \hat{p} \cdot \hat{q} \cdot N}{e^{2}(N-1)+Z_{\alpha / 2}^{2} \cdot \hat{p} \cdot \hat{q}}
$$

Considerando $\mathrm{Z}(\propto / 2)$ valor tabelado de 1,96 , percentual estimado $(\mathrm{p})$ para o evento de $0,5 \%$, complemento de $\mathrm{p}$ de zero $(\mathrm{q}=1-\mathrm{p})$ e um erro amostral $(\propto)$ de $0,05 \%$. A amostra mínima deveria ser de 131 estudantes.

Para participar do estudo, o estudante devia ter idade maior ou igual a 18 anos, estar matriculado em todas as disciplinas referentes a cada período do curso e estar frequentando regularmente há mais de 30 dias as aulas teóricas e/ou práticas. Foram excluídos do estudo aqueles que estavam afastados do curso por qualquer motivo durante a coleta de dados. Constituíram-se como perdas as recusas à participação na pesquisa e não estar presente no momento da coleta de dados.

Os dados foram coletados em dezembro de 2014, por duas autoras da pesquisa, previamente capacitadas, durante o período letivo. Os participantes foram acessados nas salas de aula, mediante autorização do professor, antes do início ou ao término da aula e, por vezes, nos intervalos das aulas. Os estudantes que aceitaram participar da pesquisa assinaram o Termo de Consentimento Livre e Esclarecido em duas vias, ficando de posse de uma delas.

Utilizou-se um questionário autoaplicado, elaborado pelos autores, com perguntas referentes ao perfil sociodemográfico (sexo, faixa etária, cor da pele autorreferida, procedência, estado civil, filhos e outra atividade além da graduação), acadêmico (semestre no curso, ter ou não bolsa de estudos, estar em atividades de extensão ou vivências* e estar em aulas práticas) e de saúde (uso de bebida alcoólica e tabaco, tempo de uso de computador, fazer atividade física, possuir tempo para lazer, meio de transporte utilizado para o

\footnotetext{
* Atividades práticas assistenciais que fazem parte de um programa de ensino e extensão da instituição em estudo, o qual se denomina: Programa de Formação Complementar em Enfermagem- PROFCEN.
} 


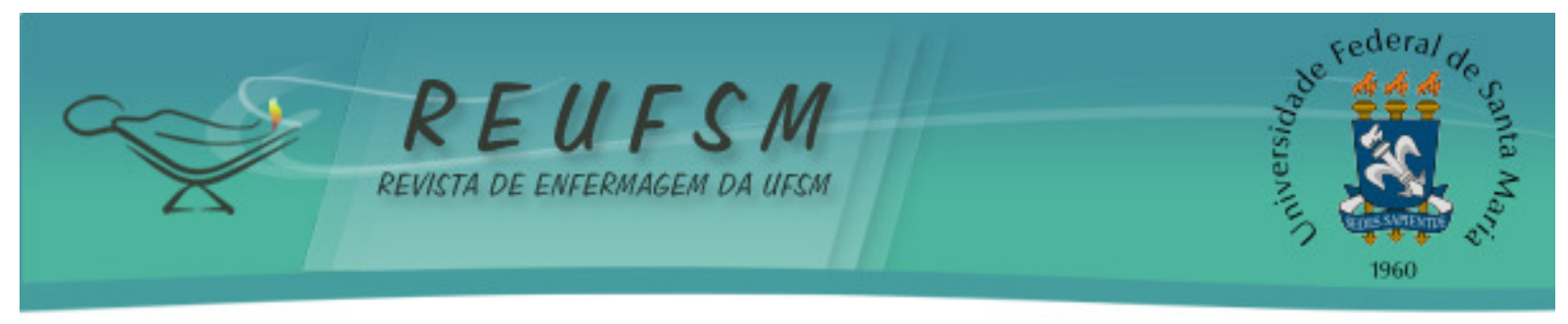

deslocamento às aulas, possuir doença diagnosticada pelo médico, se afirmativo, quantas doenças e uso de medicação contínua).

Para avaliação da suspeição de DPMs, utilizou-se a versão brasileira do Self-Reporting Questionnaire-20 (SRQ-20), ${ }^{10}$ questionário autopreenchível, que avalia a presença ou ausência de sintomas relacionados aos DPMs nos 30 dias anteriores à investigação. É composto por 20 questões dicotômicas, cada uma das alternativas tendo valor de 0 ou 1 , em que o 1 indica que os sintomas estavam presentes no último mês, e zero (0), que estavam ausentes. ${ }^{11}$ Como ponto de corte para suspeição de DPMs, adotou-se sete ou mais questões com respostas afirmativas para ambos os sexos. ${ }^{12}$

Os dados foram organizados no programa Epiinfo®, versão 6.04, com dupla digitação independente. Após a verificação de erros e inconsistências na digitação, realizou-se a análise descritiva e analítica no programa PASW Statistics® (Predictive Analytics Software, da SPSS Inc., Chicago - USA) 18.0 for Windows. Para o escore do SRQ-20 (variável quantitativa discreta) foram utilizadas as medidas de tendência central (média e mediana) e dispersão (desvio padrão e amplitude). As variáveis categóricas foram descritas pelas frequências absoluta (n) e relativa (\%). A verificação da associação entre as variáveis e os DPMs foi medida pelo teste Qui-Quadrado, ao nível de significância de 5\%.

Este estudo foi aprovado pelo Comitê de Ética em Pesquisa da Universidade Federal de Santa Maria - UFSM/RS, sob Certificado de Apresentação para Apreciação Ética $n^{\circ}$ 37866414.2.0000.5346. Foram cumpridas as exigências contidas na Resolução 466/12, de modo a garantir a voluntariedade da participação e o anonimato dos participantes.

\section{RESULTADOS}

Dos 197 estudantes matriculados, 29 foram excluídos do estudo: ser menor de 18 anos (2), estar em trancamento total do curso (11), em intercâmbio (2), em licença maternidade (1), não estavam matriculados em todas as disciplinas do respectivo período no curso (11) e participaram como coletadores da pesquisa (2). A população elegível foi de 168 estudantes. Destes, houve 19 (11,3\%) perdas (dois não aceitaram participar da pesquisa e 17 não estavam presentes nos momentos da coleta de dados). Participaram 149 (88,7\%) estudantes.

Predominaram os estudantes do sexo feminino $(88,6 \%)$, com idade entre 21 e 30 anos $(48,3 \%)$ e com cor da pele autorreferida branca $(79,2 \%)$. Quanto à procedência, prevaleceram 


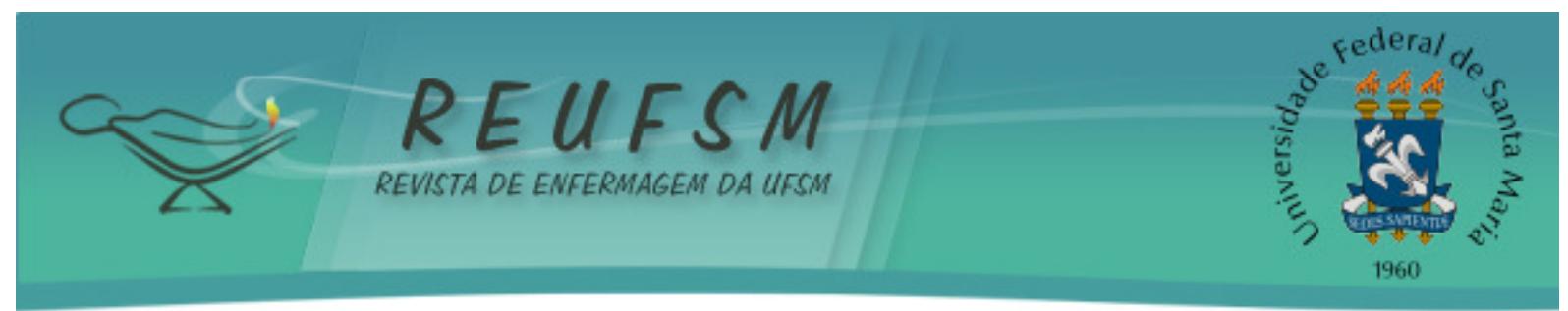

os de outra cidade $(52,3 \%)$, que eram solteiros $(84,6 \%)$, sem filhos $(88,6 \%)$ e que não possuíam outra atividade além da graduação $(87,9 \%)$.

Quanto ao perfil acadêmico, responderam à pesquisa predominantemente os estudantes do $4^{\circ}$ semestre do curso $(18,1 \%)$; que tinham algum tipo de bolsa (iniciação científica, assistência, entre outras) (55,7\%) e que não estavam em período de atividades de extensão ou vivências (91,3\%), porém em aulas práticas $(73,8 \%)$.

Em relação aos hábitos de saúde, prevaleceram estudantes que às vezes faziam uso de bebida alcoólica $(65,1 \%)$, que não eram tabagistas $(92,6 \%)$, que usavam o computador em um tempo médio de uma a três horas diárias $(64,4 \%)$, que realizavam atividade física $(38,2 \%)$, que às vezes possuíam tempo para o lazer $(49,7 \%)$ e que utilizavam ônibus para se locomover às aulas $(65,1 \%)$. No que se refere à presença doença com diagnóstico médico e uso de medicação, $79,7 \%$ afirmaram não possuir doença e 73,2\%, não fazer uso de medicação contínua.

A frequência de presença ou ausência de sintomas referentes aos DPMs, mensurada pelo SRQ-20, nos 30 dias anteriores à investigação, está descrita na Tabela 1.

Tabela 1 - Frequência das respostas dos estudantes de enfermagem, de acordo com as questões do SelfReporting Questionnaire-20 (SRQ-20). Santa Maria/RS, 2014. (N=149)

\begin{tabular}{lrrrr}
\hline \multicolumn{1}{c}{ Questão SRQ-20 } & \multicolumn{2}{c}{ Não } & \multicolumn{2}{c}{ Sim } \\
\cline { 2 - 5 } & $\mathrm{N}$ & $\mathbf{\%}$ & $\mathrm{N}$ & \% \\
\hline Tem dores de cabeça frequentemente? & 61 & 40,9 & 88 & 59,1 \\
Tem falta de apetite? & 120 & 80,5 & 29 & 19,5 \\
Dorme mal? & 57 & 38,3 & 92 & 61,7 \\
Assusta-se com facilidade? & 96 & 64,4 & 53 & 35,6 \\
Tem tremores nas mãos? & 112 & 75,2 & 37 & 24,8 \\
Sente-se nervoso, tenso ou preocupado? & 22 & 14,8 & 127 & 85,2 \\
Tem má digestão? & 106 & 71,1 & 43 & 28,9 \\
Tem dificuldade de pensar com clareza? & 92 & 61,7 & 57 & 38,3 \\
Tem se sentido triste ultimamente? & 87 & 58,4 & 62 & 41,6 \\
Tem chorado mais do que o costume? & 103 & 69,1 & 46 & 30,9 \\
Encontra dificuldade em realizar com satisfação suas atividades & 82 & 55,0 & 67 & 45,0 \\
diárias? & & & & \\
Tem dificuldade em tomar decisões? & 93 & 62,4 & 56 & 37,6 \\
Tem dificuldade no serviço, no emprego? (seu trabalho é penoso, & 133 & 89,3 & 16 & 10,7 \\
lhe causa sofrimento). & & & & \\
É incapaz de desempenhar um papel útil em sua vida? & 134 & 89,9 & 15 & 10,1 \\
Tem perdido o interesse pelas coisas? & 109 & 73,2 & 40 & 26,8 \\
Você se sente uma pessoa inútil, sem préstimo? & 136 & 91,3 & 13 & 8,7 \\
Tem tido ideia de acabar com a vida? & 144 & 96,6 & 5 & 3,4 \\
Sente-se cansado o tempo todo? & 58 & 38,9 & 91 & 61,1 \\
Tem sensações desagradáveis no estômago? & 91 & 61,1 & 58 & 38,9 \\
Você se cansa com facilidade? & 50 & 33,6 & 99 & 66,4 \\
\hline
\end{tabular}




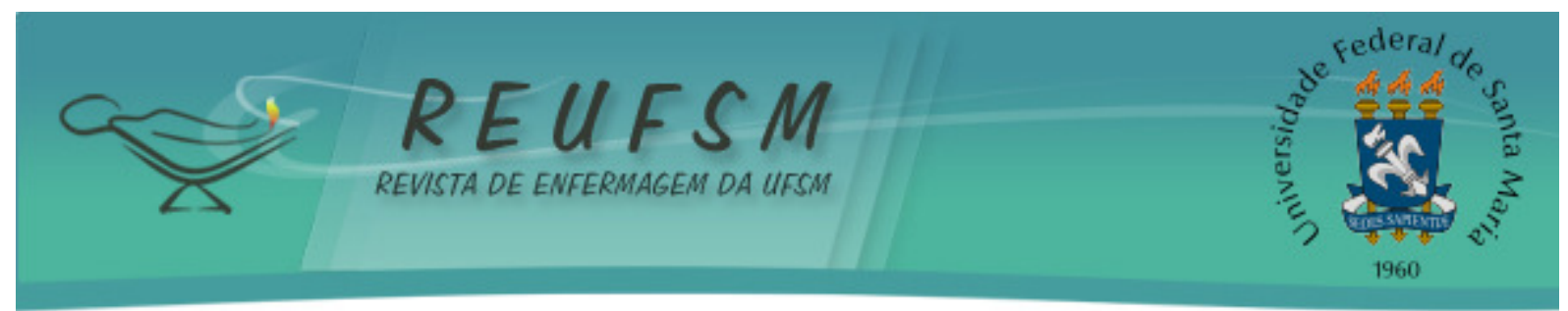

Das 20 questões do SRQ-20, as questões “Sente-se nervoso, tenso ou preocupado?", "Você se cansa com facilidade?" e "Dorme mal?" foram as que obtiveram maior frequência de respostas positivas, respectivamente. Já as questões "Você se sente uma pessoa inútil, sem préstimo?" e “Tem tido ideia de acabar com a vida?" apresentaram os percentuais mais baixos para a resposta afirmativa.

A prevalência de DPMs nos estudantes de enfermagem foi de 54,4 \% ( $\mathrm{N}=81)$. A média de respostas afirmativas foi de 7,34 $( \pm 3,76)$, mediana 7,0, mínimo zero $(0)$ e máximo 17. A Tabela 2 apresenta a relação entre o perfil sociodemográfico dos participantes e a suspeição para DPMs.

Tabela 2 - Distribuição dos estudantes de enfermagem, segundo características sociodemográficas e suspeição para Distúrbios Psíquicos Menores (DPMs). Santa Maria/RS, 2014. (N=149)

\begin{tabular}{|c|c|c|c|c|c|c|c|c|}
\hline \multirow{3}{*}{\multicolumn{2}{|c|}{ Variáveis sociodemográficas }} & \multirow{2}{*}{\multicolumn{2}{|c|}{ Frequência }} & \multicolumn{4}{|c|}{ DPMs } & \multirow{3}{*}{$\mathbf{p}$} \\
\hline & & & & \multicolumn{2}{|c|}{ Não } & \multicolumn{2}{|c|}{ Sim } & \\
\hline & & $\mathbf{N}$ & $\%$ & $\mathbf{N}$ & $\%$ & $\mathbf{N}$ & $\%$ & \\
\hline Sexo & & & & & & & & $0,094 *$ \\
\hline Feminino & & 132 & 88,6 & 57 & 43,2 & 75 & 56,8 & \\
\hline Masculino & & 17 & 11,4 & 11 & 64,7 & 6 & 35,3 & \\
\hline Faixa etária & & & & & & & & $0,705^{*}$ \\
\hline 18 a 20 anos & & 66 & 44,3 & 28 & 42,4 & 38 & 57,6 & \\
\hline 21 a 30 anos & & 72 & 48,3 & 34 & 47,2 & 38 & 52,8 & \\
\hline 31 a 46 anos & & 11 & 7,4 & 6 & 54,5 & 5 & 45,5 & \\
\hline Cor da pele autorreferida ${ }^{\dagger}$ & & & & & & & & $0,062^{\dagger}$ \\
\hline Branca & & 118 & 79,2 & 56 & 47,5 & 62 & 52,5 & \\
\hline Preto/Negra & & 14 & 9,4 & 7 & 50,0 & 7 & 50 & \\
\hline Parda & & 14 & 9,4 & 3 & 21,4 & 11 & 78,6 & \\
\hline Indígena & & 3 & 2,0 & 2 & 66,7 & 1 & 33,3 & \\
\hline Procedência & & & & & & & & $0,429 *$ \\
\hline Santa Maria & & 71 & 47,7 & 30 & 42,3 & 41 & 57,7 & \\
\hline Outras cidades & & 78 & 52,3 & 38 & 48,7 & 40 & 51,3 & \\
\hline Situação conjugal & & & & & & & & $0,497 *$ \\
\hline Casado/união estável & & 23 & 15,4 & 12 & 52,2 & 11 & 47,8 & \\
\hline Solteiro & & 126 & 84,6 & 56 & 44,4 & 70 & 55,6 & \\
\hline Filhos & & & & & & & & $0,901 *$ \\
\hline Não & & 132 & 88,6 & 60 & 45,5 & 72 & 54,5 & \\
\hline Sim & & 17 & 11,4 & 8 & 47,1 & 9 & 52,9 & \\
\hline $\begin{array}{l}\text { Outra atividade além } \\
\text { graduação }\end{array}$ & da & & & & & & & $0,540^{*}$ \\
\hline Não & & 131 & 87,9 & 61 & 46,6 & 70 & 53,4 & \\
\hline Sim & & 18 & 12,1 & 7 & 38,9 & 11 & 61,1 & \\
\hline
\end{tabular}

*Teste Qui-quadrado. ${ }^{\dagger}$ Teste Qui-quadrado com correção (caselas com menos de 5).

$\mathrm{Na}$ avaliação da relação entre as variáveis sociodemográficas e suspeição para DPMs, não foram evidenciadas diferenças estatísticas significativas entre os grupos $(p>0,05)$. A Tabela 3 apresenta os dados referentes ao perfil acadêmico dos participantes, de acordo com a presença ou ausência de suspeição para DPMs. 


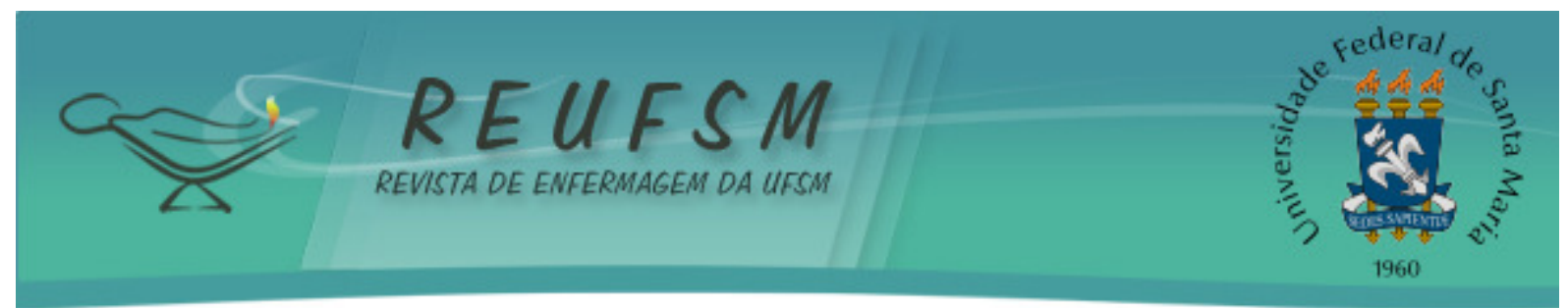

Tabela 3 - Distribuição dos estudantes de enfermagem, segundo o perfil acadêmico e suspeição para Distúrbios Psíquicos Menores (DPMs). Santa Maria/RS, 2014. (N=149)

\begin{tabular}{|c|c|c|c|c|c|c|c|}
\hline \multirow{3}{*}{ Variáveis acadêmicas } & \multirow{2}{*}{\multicolumn{2}{|c|}{ Frequência }} & \multicolumn{4}{|c|}{ DPMs } & \multirow{3}{*}{$\mathbf{p}^{*}$} \\
\hline & & & \multicolumn{2}{|c|}{ Não } & \multicolumn{2}{|c|}{ Sim } & \\
\hline & $\mathrm{N}$ & $\%$ & $\mathrm{~N}$ & $\%$ & $\mathrm{~N}$ & $\%$ & \\
\hline Semestre no curso & & & & & & & 0,424 \\
\hline 1 & 16 & 10,7 & 5 & 31,3 & 11 & 68,8 & \\
\hline 2 & 23 & 15,4 & 9 & 39,1 & 14 & 60,9 & \\
\hline 3 & 15 & 10,1 & 8 & 53,3 & 7 & 46,7 & \\
\hline 4 & 27 & 18,1 & 13 & 48,1 & 14 & 51,9 & \\
\hline 5 & 18 & 12,1 & 6 & 33,3 & 12 & 66,7 & \\
\hline 6 & 21 & 14,1 & 14 & 66,7 & 7 & 33,3 & \\
\hline 7 & 14 & 9,4 & 6 & 42,9 & 8 & 57,1 & \\
\hline 8 & 15 & 10,1 & 7 & 46,7 & 8 & 53,3 & \\
\hline Possui bolsa (IC, assistência) & & & & & & & 0,711 \\
\hline $\operatorname{Sim}$ & 83 & 55,7 & 39 & 47,0 & 44 & 53,0 & \\
\hline Não & 66 & 44,3 & 29 & 43,9 & 37 & 56,1 & \\
\hline Atividades de extensão ou vivências & & & & & & & 0,228 \\
\hline Sim & 13 & 8,7 & 8 & 61,5 & 5 & 38,5 & \\
\hline Não & 136 & 91,3 & 60 & 44,1 & 76 & 55,9 & \\
\hline Em aulas práticas & & & & & & & 0,155 \\
\hline Sim & 110 & 73,8 & 54 & 49,1 & 56 & 50,9 & \\
\hline Não & 39 & 26,2 & 14 & 35,9 & 25 & 64,1 & \\
\hline
\end{tabular}

*Teste Qui-Quadrado.

Na avaliação da associação entre as variáveis acadêmicas e DPMs, não foi evidenciada diferença estatística significativa entre os grupos ( $>00,05)$. A Tabela 4 apresenta os dados referentes ao perfil de saúde dos participantes, de acordo com a presença ou ausência de suspeição para DPMs.

Tabela 4 - Distribuição dos estudantes de enfermagem, segundo perfil de saúde e suspeição para Distúrbios Psíquicos Menores (DPMs). Santa Maria/RS, 2014. (N=149)

\begin{tabular}{|c|c|c|c|c|c|c|c|}
\hline \multirow{3}{*}{ Variáveis de saúde } & \multirow{2}{*}{\multicolumn{2}{|c|}{ Frequência }} & \multicolumn{4}{|c|}{ DPMs } & \multirow{3}{*}{$\mathbf{p}$} \\
\hline & & & \multicolumn{2}{|c|}{ Não } & \multicolumn{2}{|c|}{ Sim } & \\
\hline & $\mathrm{N}$ & $\%$ & $\mathrm{~N}$ & $\%$ & $\mathrm{~N}$ & $\%$ & \\
\hline Uso de bebida alcoólica & & & & & & & $0,956^{*}$ \\
\hline Sim & 13 & 8,7 & 6 & 46,2 & 7 & 53,8 & \\
\hline Às Vezes & 97 & 65,1 & 45 & 46,4 & 52 & 53,6 & \\
\hline Não & 39 & 26,2 & 17 & 43,6 & 22 & 56,4 & \\
\hline Uso de tabaco & & & & & & & $0,302^{\dagger}$ \\
\hline Sim & 5 & 3,4 & 1 & 20,0 & 4 & 80,0 & \\
\hline Às Vezes & 6 & 4,0 & 4 & 66,7 & 2 & 33,3 & \\
\hline Não & 138 & 92,6 & 63 & 45,7 & 75 & 54,3 & \\
\hline Atividade física & & & & & & & $0,292^{*}$ \\
\hline Sim & 57 & 38,2 & 30 & 52,6 & 27 & 47,4 & \\
\hline Às vezes & 56 & 37,6 & 25 & 44,6 & 31 & 55,4 & \\
\hline Não & 36 & 24,2 & 13 & 36,1 & 23 & 63,9 & \\
\hline Tempo para lazer & & & & & & & $0,064^{\dagger}$ \\
\hline Sim & 68 & 45,6 & 38 & 55,9 & 30 & 44,1 & \\
\hline Às vezes & 74 & 49,7 & 28 & 37,8 & 46 & 62,2 & \\
\hline Não & 7 & 4,7 & 2 & 28,6 & 5 & 71,4 & \\
\hline Meio de transporte & & & & & & & $0,211^{\dagger}$ \\
\hline
\end{tabular}




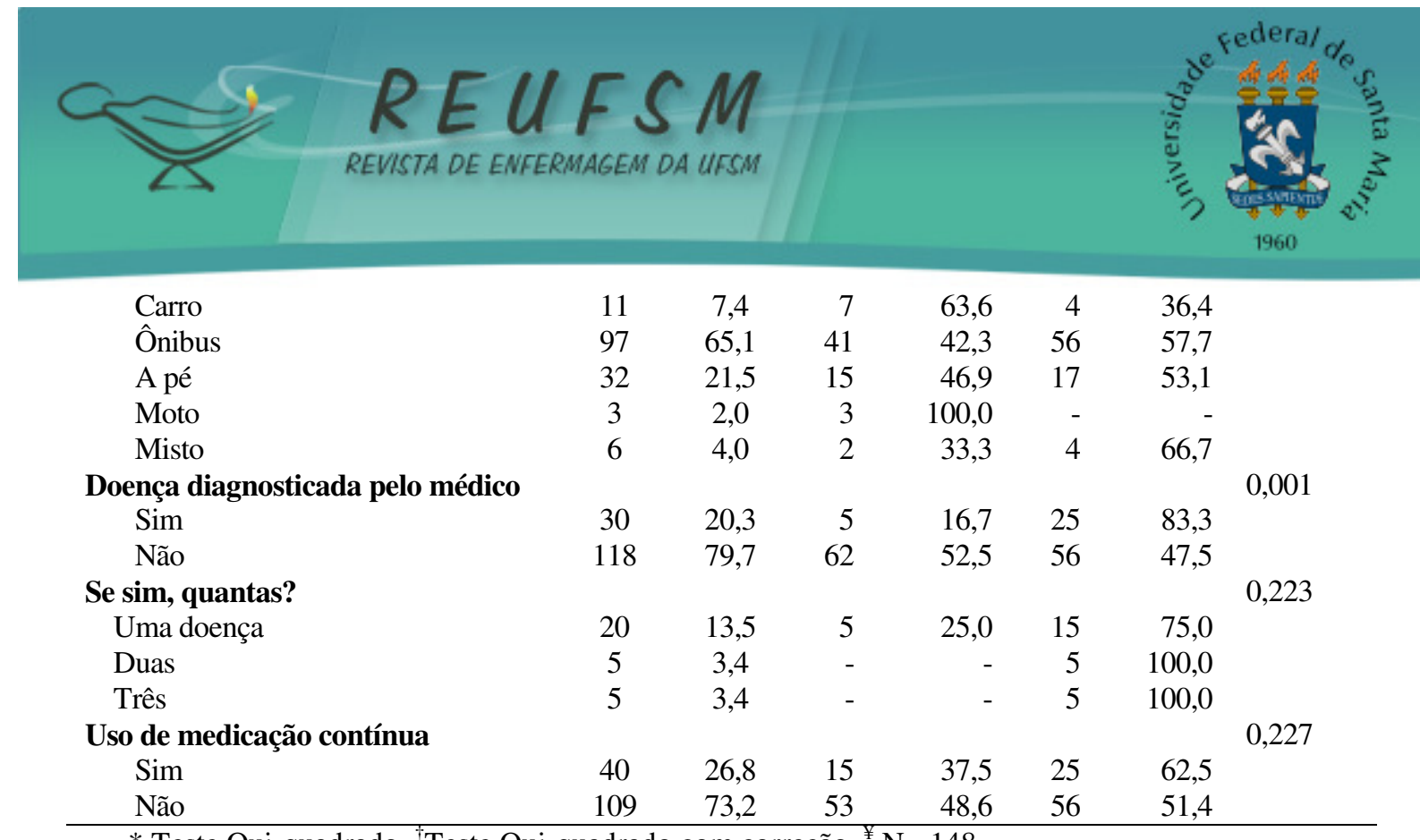

* Teste Qui-quadrado. "Teste Qui-quadrado com correção. ${ }^{¥} \mathrm{~N}=148$.

Os estudantes com presença de doença com diagnóstico médico tiveram significativamente maior percentual de suspeição para DPMs $(\mathrm{p}<0,05)$.

\section{DISCUSSÃO}

A elevada prevalência de DPMs evidenciada neste estudo $(54,4 \%)$ difere da observada em duas pesquisas realizadas recentemente em cursos de enfermagem das Regiões Norte e Sul do Brasil. Nesses estudos, observaram-se prevalências de $22,1 \%{ }^{13}$ e $26 \% .{ }^{14}$ Em acadêmicos de medicina os percentuais aproximam-se aos deste estudo (46,9\% e 33,6\%). ${ }^{15-16}$ Em cursos de educação física, nutrição, biologia, psicologia, fisioterapia, entre outros, os percentuais são inferiores, entre $9,5 \%$ e $40 \% .^{14,17}$

Depreende-se disso que, embora existam proporções diferentes de DPMs em alguns grupos de estudantes, a presença de estressores é real e pode ser própria da vida acadêmica. No ensino superior, a responsabilidade e pontualidade exigidas com as atividades acadêmicas, como os prazos estipulados para relatórios e trabalhos, geram preocupações, as quais podem favorecer a presença dos DPMs. Além disso, enfatiza-se que, por a fase de coleta de dados ter ocorrido no término do semestre letivo, acredita-se que os estudantes encontravam-se em um momento de exaustão física e psíquica, pela demanda de atividades acadêmicas, como a realização das provas finais, entrega de trabalhos acadêmicos e a finalização de aulas práticas e estágios em serviços de saúde, fatores estes que contribuem com o esgotamento psíquico.

No que tange à frequência das respostas afirmativas ao SRQ-20 (dor de cabeça, dormir mal e sentir-se nervoso e cansado), resultado semelhante foi observado em estudo que avaliou os riscos psicossociais entre acadêmicos de enfermagem de uma faculdade pública do Rio de Janeiro, nas 


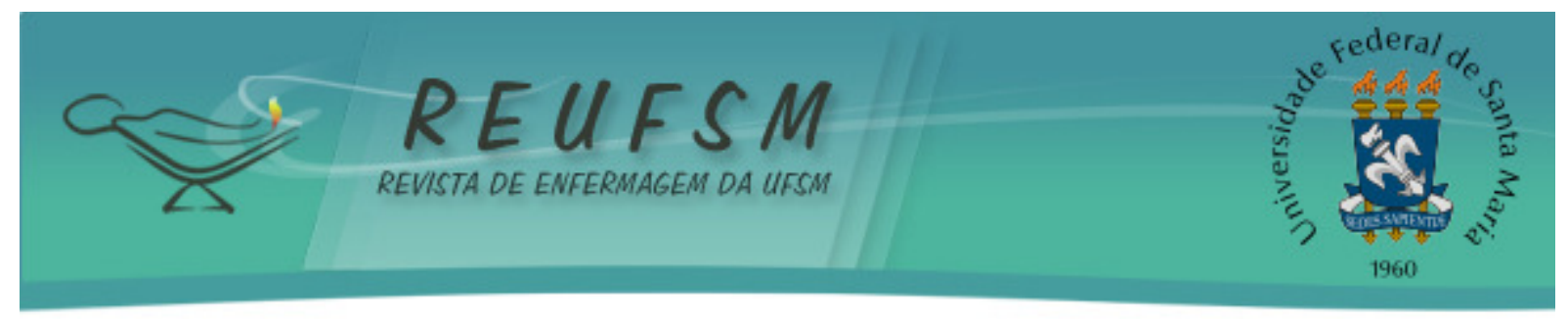

questões relacionadas à cefaleia frequente $(56,7 \%)$ e ao nervosismo/preocupação $(83,8 \%)$. Já os demais itens, como padrão de sono ruim $(89,1 \%)$, cansar-se com facilidade $(86,4 \%)$ e sentir-se cansado o tempo todo $(78,4 \%)$, apresentaram achados superiores ao desta pesquisa. ${ }^{18} \mathrm{Na}$ Universidade Federal do Acre, em estudo com a mesma população, os percentuais de respostas afirmativas para as mesmas questões do SRQ-20 foram menores, variando de $18,4 \%$ a $31,6 \%{ }^{19}$

Ressalta-se que 3,4\% ( $\mathrm{N}=5)$ dos participantes deste estudo assinalaram resposta positiva na questão referente ao pensamento suicida (“tem tido ideia de acabar com a vida?"). De acordo com a literatura, ${ }^{20}$ existe um significativo número de suicídios entre estudantes universitários e o estresse, a depressão e o uso de álcool e drogas constituem-se como fatores de risco. Nesse sentido, a identificação precoce é importante e merece a devida atenção. $O$ acompanhamento psicológico ou psiquiátrico, dependendo do caso, podem auxiliar na redução ou até na eliminação do risco de suicídio.

Em relação às características sociodemográficas, os resultados deste e de outros estudos desenvolvidos com estudantes de medicina e de enfermagem não evidenciaram relação significativa para DPMs. ${ }^{14,16}$ No contexto do perfil acadêmico, apesar de não haver diferença estatística significativa entre os grupos, observa-se elevada prevalência de DPMs nos estudantes do primeiro semestre $(68,8 \%)$. Em estudantes de biologia, enfermagem, psicologia e fisioterapia da Faculdade Anhanguera, em Rio Grande/RS, 44\% dos acadêmicos do início da graduação apresentaram suspeição de DPMs. ${ }^{17}$

Neste universo, destaca-se que o ingresso na graduação, para muitos indivíduos, geralmente ocorre quando estão em transição da adolescência para a avida adulta. Assim, a entrada na universidade pode ser acompanhada de dúvidas e incertezas, visto que os estudantes talvez ainda não possuam uma real noção da profissão que escolheram. Podem então enfrentar certa pressão psicológica, já que o mesmo, ainda habituado com o ensino mecanicista do nível médio, se depara com o contexto reflexivo e pensante do ensino superior. ${ }^{1}$

Junto a isso, a prática profissional ainda insuficiente, somada às possíveis dificuldades de relacionamento com novos colegas e professores, pode gerar quadros de ansiedade e estresse. Também, as novas condições de transporte e moradia, que para alguns é longe dos pais, podem interferir na saúde psíquica. Com isso, observa-se que esse período pode envolver situações que tragam potenciais repercussões para a saúde psíquica do estudante. ${ }^{1}$ 


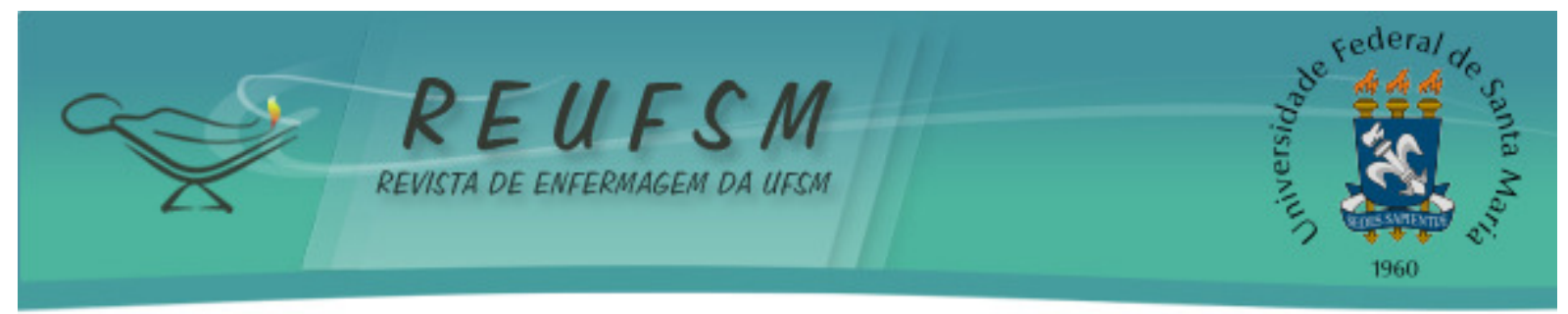

No curso de enfermagem da instituição estudada, as atividades referentes ao primeiro período envolvem altas cargas horárias de aulas teóricas que, associadas à nova rotina de estudos e atividades pessoais, podem desencadear adoecimento psíquico. Também, ao iniciarem a graduação, os estudantes ficam distantes da realidade da enfermagem, já que, nos primeiros semestres, a maior parte dos conteúdos é ministrada por professores de formação distinta e em conjunto com outros cursos, por serem comuns entre os da área da saúde. Em novas propostas curriculares, a carga horária, o número e o formato das disciplinas são levados em consideração pela comunidade acadêmica.

Quanto aos percentuais de suspeição de DPMs nos estudantes do quinto período da graduação em enfermagem, há que se considerar que nesse momento do curso eles enfrentam novos desafios ao vivenciarem os primeiros contatos com pacientes em situações críticas de vida no ambiente hospitalar. É nesta fase que eles conhecem algumas das unidades hospitalares destinadas às práticas de cuidado mais complexas, como a Unidade de Terapia Intensiva Adulto e Bloco Cirúrgico. Tal situação pode possibilitar uma mistura de anseios e medos.

No que se refere aos estudantes com ou sem bolsa, a suspeição de DPMs prevaleceu entre aqueles que não possuíam. Em graduandos de medicina da Universidade Federal do Sergipe, pesquisa identificou sintomas depressivos leves a moderados entre aqueles que possuíam renda familiar mais baixa. ${ }^{21}$ As dificuldades financeiras podem ser fatores agravantes de sofrimento psíquico no estudante. Neste sentido, as bolsas de auxílio, indiferente do tipo, são importantes, principalmente para aqueles que necessitam de aporte financeiro para subsidiar sua formação.

Em relação às atividades de extensão, a prevalência de DPMs foi elevada entre os estudantes que não as faziam (55,9\%). Assim, há que se considerar que as atividades de extensão podem proporcionar o sentimento de realização profissional, visto que por meio delas ele se aproxima com as atividades inerentes à profissão. Estas promovem a construção do conhecimento, contribuindo assim com a autonomia e confiança nas atividades realizadas como futuro enfermeiro.

Referente ao perfil de saúde dos estudantes, a presença de doença com diagnóstico médico apresentou significância estatística para DPMs, diferente de outro estudo que não evidenciou diferença entre os grupos avaliados. ${ }^{13}$ Dos estudantes com doenças diagnosticadas, predominou a presença de uma doença com diagnóstico $(13,4 \%)$. Entretanto, observaram-se altos percentuais de DPMs naqueles que assinalaram ter duas e três doenças com diagnóstico 


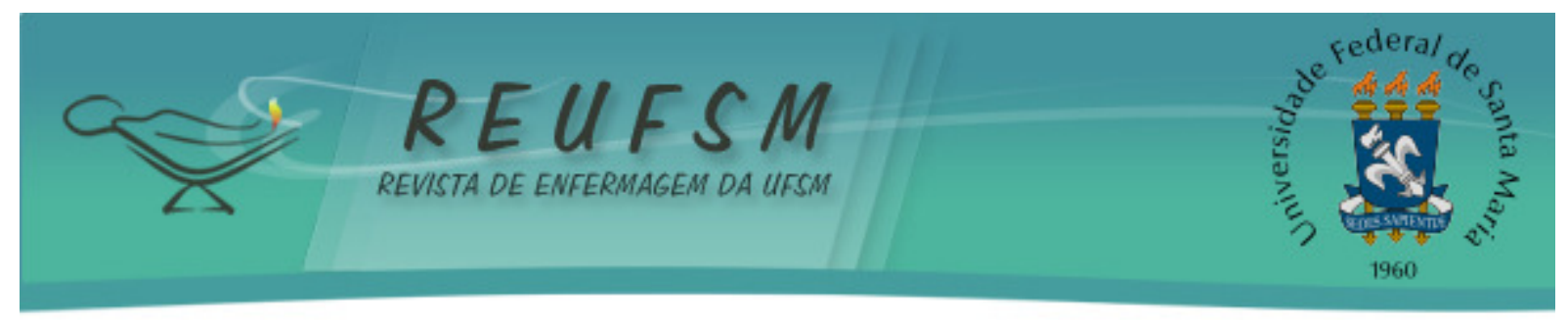

médico. Diante disso, pode-se considerar a possível relação de problemas psíquicos com a etiologia e progressão de doenças físicas. ${ }^{22}$ Em estudo realizado com enfermeiros da cidade de Pelotas/RS, evidencia-se a presença de transtornos depressivos, entre outras doenças crônicas, naqueles que se autoavaliaram com saúde ruim/razoável. ${ }^{23}$

Quando avaliados sobre o uso de medicação contínua, predominaram os estudantes que não faziam uso $(73,2 \%)$. Porém, verificou-se a presença de DPMs em $62,5 \%$ daqueles que necessitavam da terapia farmacológica contínua, o que pode estar relacionado ao fato de que parte dos usuários de medicação contínua é portadora de doenças com diagnóstico médico. Sabe-se que, geralmente, o adoecimento do corpo físico vem acompanhado dos sintomas psíquicos.

\section{CONCLUSÃO}

Mais da metade dos estudantes $(54,4 \%)$ apresentaram suspeição para DPM. Tal evidência assinala para necessidade de oferta de apoio psicológico aos mesmos, principalmente, àqueles que se encontram no início da graduação. Das variáveis aqui avaliadas, ter doença diagnosticada pelo médico apresentou relação significativa com DPM.

Neste sentido, é fundamental que o curso de graduação pesquisado identifique estratégias para promoção da saúde mental dos estudantes. Por exemplo: realização de acolhimento aos ingressantes com atividades lúdicas, como as em grupo e rodas de conversa, esclarecendo as dúvidas existentes em relação à futura profissão e às possíveis demandas acadêmicas; realização de visitas a instituições e serviços de saúde, como no próprio hospitalescola da instituição. Talvez, essas estratégias auxiliem na redução do adoecimento psíquico dos estudantes, uma vez que familiariza o estudante com a profissão escolhida.

Como limitações, o presente estudo restringe-se aos achados de um estudo transversal, o que não possibilita estabelecer relações de causa e efeito (viés da causalidade reversa). Entretanto, os resultados podem subsidiar o desenvolvimento de ações promotoras de saúde mental tanto pela coordenação do curso pesquisado, como pelos órgãos da Universidade (a Reitoria e Pró-Reitoria de Assuntos Estudantis). Podem, também, instrumentalizar as demais comunidades acadêmicas (outras instituições) na construção de programas de apoio psicológico aos estudantes e na realização de novas pesquisas sobre a temática. Nesse sentido, os achados do estudo apontam para a importância do desenvolvimento de pesquisas longitudinais e de intervenção sobre a temática. 


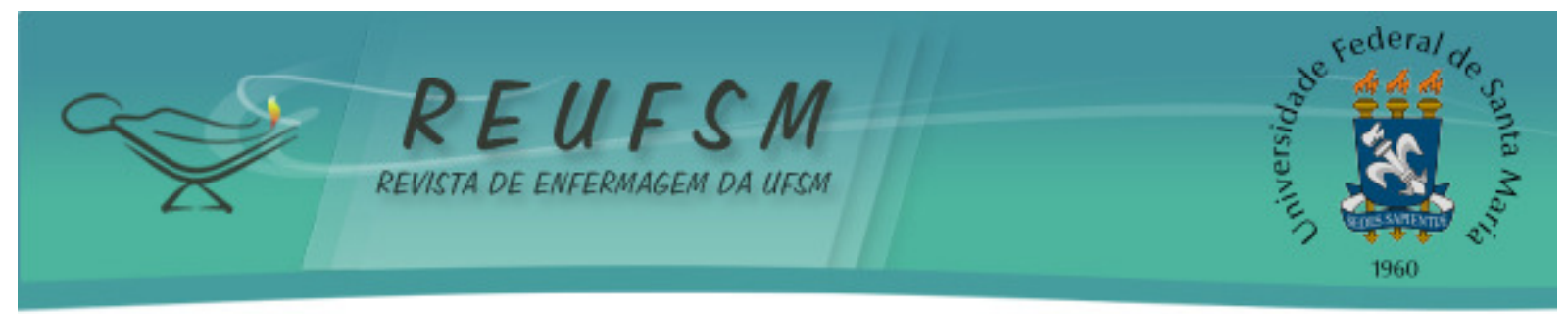

\section{REFERÊNCIAS}

1. Lima JRN, Pereira AKAM, Nascimento EGC, Alchieri JC. Percepção do acadêmico de enfermagem sobre o seu processo de saúde/doença durante a graduação. Saúde transform soc. [Internet]. 2013 [acesso em 2016 nov 28];4(4):54-62. Disponível em: http://pepsic.bvsalud.org/scielo.php?script=sci_arttext\&pid=S2178-70852013000400010.

2. Hirsch CD, Barlem ELD, Tomaschewski-Barlem JGT, Lunard VL, Oliveira ACC. Preditores do estresse e estratégias de coping utilizadas por estudantes de enfermagem. Acta Paul Enferm. 2015;28(3):224-9.

3. Canalli RTC, Moriya TM, Hayashida M. Prevenção de acidentes com material biológico entre estudantes de enfermagem. Rev Enferm UERJ. 2011;9(11):100-6.

4. Goldberg D, Huxley P. Common mental disorders: a bio-social model. London: Tavistock; 1992.

5. Alves AP, Pedrosa LAK, Coimbra MAR, Miranzi MAS, Hass VJ. Prevalência de transtornos mentais comuns entre profissionais de saúde. Rev Enferm UERJ. 2015;23(1):64-9.

6. Urbanetto JS, Magalhães MCC, Maciel VO, Sant'Anna VM, Gustavo AS, Poli-de-Figueiredo $\mathrm{CE}$, et al. Estresse no trabalho segundo o Modelo Demanda- Controle e distúrbios psíquicos menores em trabalhadores de enfermagem. Rev Esc Enferm USP. 2013;47(3):1186-93.

7. Rodrigues EP, Rodrigues US, Oliveira LMM, Laudano RCS, Nascimento CL. Prevalência de transtornos mentais comuns em trabalhadores de enfermagem em um hospital da Bahia. Rev Bras Enferm. 2014;67(2):296-301.

8. Tavares JP, Beck CLC, Magnago TSBS, Greco PBT, Prestes FC, Silva RM. Produção científica sobre os distúrbios psíquicos menores a partir do self report questionnarie. Rev Enferm UFSM [Internet]. 2011[acesso em 2016 nov 28];1(1):113-23. Disponível em: https://periodicos.ufsm.br/reufsm/article/view/2091/1518.

9. Lopes LFD. Estatística e qualidade \& produtividade. [s.d.][acesso em 2017 out 10]. Disponível em: http://felipelopes.com/CalculoAmostra.php.

10. Mari JJ, Williams P. Avalidity study of a psychiatric screening questionnaire (SQR-20) in primary care in the city of São Paulo. Br J Psychiatry. 1986;148:23-6.

11. World Health Organization (WHO). Divison of Mental Health. A user's guide to the selfreporting questionnaire (SRQ). Division of mental health world health organization [Internet].

Geneva: Switzerland; 1994 [acesso em 2016 nov 28]. Disponivel em: http://apps.who.int/iris/bitstream/10665/61113/1/WHO_MNH_PSF_94.8.pdf.

12. Gonçalves DM, Stein AT, Kapczinski F. Avaliação de desempenho do Self-Reporting Questionnaire como instrumento de rastreamento psiquiátrico: um estudo comparativo com o Structured Clinical Interview for DSM-IV-TR Cad Saúde Pública [Internet]. 2008 [acesso em 2016 nov 28];24(2):380-90. Disponível em: http://www.scielo.br/pdf/csp/v24n2/16.pdf.

13. Silva AO, Cavalcanti Neto JL. Associação entre níveis de atividade física e transtorno mental comum em estudantes universitários. Motricidade [Internet]. 2014 [acesso em 2016 nov 28];10(1):49-59. Disponível em: http://docplayer.com.br/9322703-Associacao-entreniveis-de-atividade-fisica-e-transtorno-mental-comum-em-estudantes-universitarios.html. 


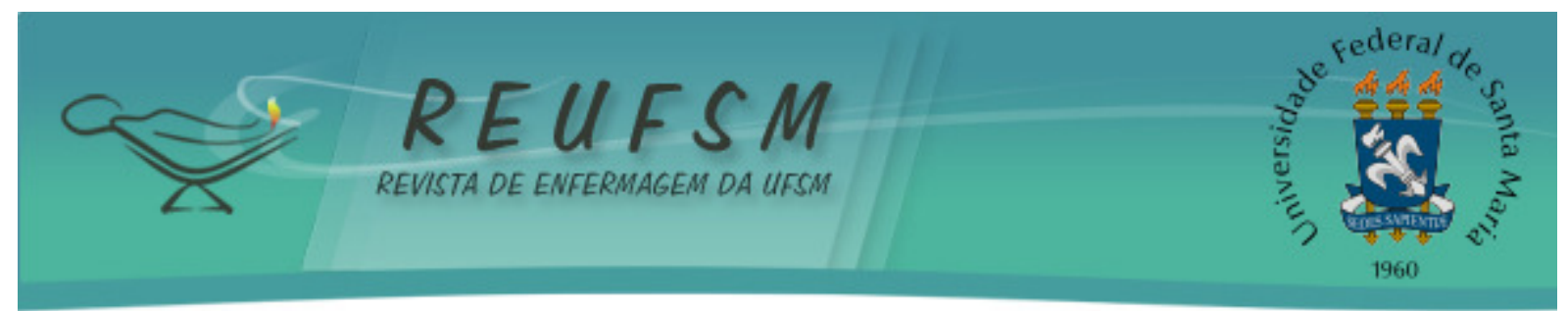

14. Souza LM, Oliveira EL, Pinheiro IS. Distúrbios psiquiátricos menores em acadêmicos de enfermagem. Rev Enferm UFPE Online [Internet]. 2014 [acesso em 2016 nov 28];8(12):4320-9. Disponível em: http://www.revista.ufpe.br/revistaenfermagem/index.php/revista/article/view/6453/pdf_6762.

15. Silva AG, Cerqueira ATAR, Lima MCP. Social support and common mental disorder among medical students. Rev Bras Epidemiol. 2014;17(1):229-42.

16. Rocha ES, Sassi AP. Transtornos mentais menores entre estudantes de medicina. Rev Bras Educ Méd. 2013;37(2):210-6.

17. Silva RS, Costa LA. Prevalência de transtornos mentais comuns entre estudantes universitários da área da saúde. Encontro Rev Psicol [Internet]. 2012 [acesso em 2016 nov 28];15(23):105-12. Disponível em: http://www.pgsskroton.com.br/seer/index.php/renc/article/view/2473/2369.

18. Oliveira EB, Costa SLT, Guimarães NSL. O trabalho do acadêmico de enfermagem no hospital geral: riscos psicossociais. Rev Enferm UERJ. 2012;20(3):317-22.

19. Silva BP, Corradi-Webster CM, Donato ECSG, Hayashida M, Siqueira MM. Transtornos mentais comuns e consumo de bebida alcoólica e tabaco entre estudantes de enfermagem de uma universidade pública na Amazônia ocidental brasileira. SMAD, Rev Eletrônica Saúde Mental Alcool Drog [Internet]. 2014 [acesso em 2016 nov 28];10(2):93-100. Disponível em: http://www.revistas.usp.br/smad/article/view/98724.

20. Dutra E. Suicídio de universitários: o vazio existencial de jovens na contemporaneidade. Estud Pesqui Psicol. 2012;12(3):924-37.

21. Costa EFO, Santana YS, Santos ATRA, Martins, LAN, Melo EV, Andrade TM. Sintomas depressivos entre internos de medicina em uma universidade pública brasileira. Rev Assoc Med Bras. 2012;58(1):53-9.

22. Gonçalves DVC, Brito LC, Carvalho MF, Sampaio CA. Percepção sobre o adoecimento entre estudantes de cursos da área da saúde. Rev Bras Educ Méd. 2015;39(1):102-11.

23. Porto AR, Rodrigues SS, Joner LR, Noguez PT, Thofehrn MB, Pai DD. Autoavaliação de saúde e doenças crônicas entre enfermeiros de Pelotas/RS. Rev Eletrônica Enferm [Internet]. 2013 [acesso em 2016 nov 28];15(3):763-71. Disponível em: https://www.fen.ufg.br/fen_revista/v15/n3/pdf/v15n3a19.pdf.

Data de submissão: 12/12/2016

Data de aceite: 16/10/2017

Autor correspondente: Cecília Mariane Pinheiro Pedro

Endereço: Universidade Federal de Santa Maria, Programa de Pós-Graduação em Enfermagem, prédio 26, sala 1302, Centro de Ciências da Saúde. Santa Maria/RS.

CEP: 97105-900

E-mail: cecilia-mariane@ hotmail.com

\section{AGRADECIMENTOS}

Programa Institucional de Bolsas de Iniciação Científica/Fundação de Amparo à Pesquisa do Estado do Rio Grande do Sul (PROBIC/FAPERGS). 